\title{
microRNA-23a promotes cell growth and metastasis in gastric cancer via targeting SPRY2-mediated ERK signaling
}

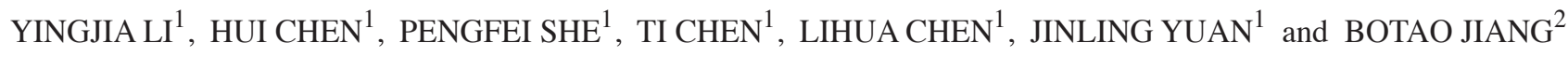 \\ ${ }^{1}$ Clinical Laboratory, The Third Xiangya Hospital, Central South University, Changsha, Hunan 410013; \\ ${ }^{2}$ Department of Neurology, The First Hospital of Changsha, Changsha, Hunan 410005, P.R. China
}

Received April 3, 2017; Accepted December 7, 2017

DOI: $10.3892 / 01.2018 .8374$

\begin{abstract}
RNAs (miRs) serve important roles in various human cancer types. Recently, miR-23a has been indicated as an oncogene in gastric cancer, but the underlying mechanism remains unclear. In the present study, reverse transcription-quantitative polymerase chain reaction and western blot analysis was used to explore the effects of miR-23a in gastric cancer. Additionally, cell proliferation, migration and invasion were examined using an MTT assay, wound healing assay and Transwell assay, respectively. Furthermore, a luciferase reporter gene assay was used to confirm the target association. It was determined that miR-23a was significantly upregulated in gastric cancer tissues and cell lines compared with adjacent tissues, and a normal gastric epithelial cell line. Furthermore, its upregulation was significantly associated with cancer progression and poor prognosis of patients. Knockdown of miR-23a caused a notable reduction in the proliferation, migration and invasion of gastric cancer AGS cells. Sprouty homolog 2 (SPRY2) was then predicted to be target gene of miR-23a. A luciferase reporter gene assay data demonstrated that miR-23a has the ability to directly bind to the 3'-untranslational region of SPRY2 mRNA. Further investigation demonstrated that SPRY2 was significantly downregulated in gastric cancer tissues and cell lines, and the protein expression of SPRY2 was negatively regulated by miR-23a in AGS cells. Furthermore, knockdown of SPRY2 reduced the suppressive effects of miR-23a inhibition in AGS cell proliferation, migration and invasion. In addition, the activity of extracellular signal-regulated kinase (ERK) signaling was also inhibited by the miR-23a/SPRY2 knockdown in AGS cells. The present study indicated that miR-23a serves a promoting role in gastric cancer via targeting SPRY2 and downstream ERK signaling.
\end{abstract}

Correspondence to: Professor Hui Chen, Clinical Laboratory, The Third Xiangya Hospital, Central South University, 138 Tongzipo Road, Changsha, Hunan 410013, P.R. China

E-mail: xiangyachenhui2017@163.com

Key words: gastric cancer, microRNA, sprouty homolog 2, extracellular signal-regulated kinase

\section{Introduction}

Gastric cancer is one of the most common human cancer types $(1,2)$. Due to the high rate of recurrence and metastasis, patients with gastric cancer have demonstrated a poor 5-year survival time $(1,2)$. Although there have been great improvements in cancer diagnosis and treatment in recent years, gastric cancer still remains among the top five for cancer-associated mortality globally (1-3). Revealing the molecular mechanism underlying gastric cancer development and progression may assist in developing more effective therapeutic strategies against gastric cancer.

microRNAs (miRs) are a type of small non-coding RNAs containing 22-25 nucleotides, and act as key regulators for gene expression via directly binding the mRNA 3'-untranslated region (UTR) of their target genes, which leads to translation inhibition or mRNA degradation $(4,5)$. Through these functions, miRs participate in the regulation of various biological processes, including cell development and differentiation, angiogenesis, cell survival, proliferation, cell cycle progression, migration, invasion and tumorigenesis $(6,7)$. In recent years, numerous miR-targeting oncogenes and tumor suppressors have been identified in numerous human cancer types (8-10). Through inhibiting the expression of these oncogenes and/or tumor suppressors, these miRs serve suppressive or promoting roles in tumor growth, and metastasis $(11,12)$.

miR-23a has been demonstrated to serve different roles in various human cancer types $(13,14)$. It functions as a tumor suppressor in osteosarcoma, and the ectopic expression of miR-23a led to reduced proliferation, migration and invasion of osteosarcoma cells (14). Additionally, miR-23a inhibits epithelial-mesenchymal transition (EMT) in endometrial endometrioid adenocarcinoma by targeting mothers against decapentaplegic homolog 3 (13). On the contrary, miR-23a was identified as an oncogene in gastric cancer $(15,16)$, promoting the growth of gastric adenocarcinoma cell line MGC803 and downregulating the interleukin-6 receptor (15). miR-23a inhibits PPP2R5E expression, which facilitates the growth of gastric cancer cells (16); however, the exact regulatory mechanism underlying miR-23a in gastric cancer remains unclear.

Sprouty homolog 2 (SPRY2) is a member of the sprouty family and inhibits the activity of receptor tyrosine kinase signaling, which is required for the growth factor-stimulated 
translocation of the protein to membrane ruffles $(17,18)$. Previous studies have indicated that SPRY2 serves a suppressive role in different human cancer types (19-21); however, the regulatory mechanism underlying SPRY2 expression in gastric cancer remains unknown.

In the present study, the aim was to explore the molecular mechanism underlying miR-23a gastric cancer growth and metastasis.

\section{Materials and methods}

Tissue collection. The present study was approved by the Ethical Committee of The Third Xiangya Hospital (Changsha, China). A total of 78 gastric cancer tissues, as well as the adjacent non-tumor tissues were obtained from The Third Xiangya Hospital between January 2010 and March 2011. These patients with gastric cancer included 45 male and 33 female aged between $43-83$ years, with a mean of 67.4 years. Written informed consent was obtained from all patients. All tissue samples were confirmed using histopathological evaluation and stored at $-80^{\circ} \mathrm{C}$ until further use. The clinical information for patients with gastric cancer is summarized in Table I.

Reverse transcription-quantitative polymerase chain reaction $(R T-q P C R)$. Total RNA was extracted using the TRIzol ${ }^{\circledR}$ reagent (Thermo Fisher Scientific, Inc., Waltham, MA, USA), which was then converted to cDNA using a Taqman ${ }^{\circledR}$ miRNA Reverse Transcription kit (Thermo Fisher Scientific, Inc.), according to the manufacturer's protocol. The expression of miR was determined using a Hairpin-it miRNAs qPCR Quantitation kit (Shanghai GenePharma Co, Ltd., Shanghai, China). U6 was used as the internal reference. The expression of mRNA was examined using a SYBR ${ }^{\circledR}$ Green qPCR Assay kit (Thermo Fisher Scientific, Inc.), according to the manufacturer's protocol. GAPDH was used as the internal reference for mRNA. The thermocycling conditions were initial denaturation at $95^{\circ} \mathrm{C}$ for $10 \mathrm{~min}$, then 40 cycles at $95^{\circ} \mathrm{C}$ for $15 \mathrm{sec}$ and annealing/elongation at $60^{\circ} \mathrm{C}$ for $15 \mathrm{sec}$. The relative expression was analyzed by the $2^{-\Delta \Delta \mathrm{Cq}}$ method (22). The primers for miR-23a and U6, which was used as the internal reference for microRNAs, were purchased from Fulgene (Guangzhou, China; cat nos. HmiRQP0345 and HmiRQP9001, respectively). The primer sequences were as follows: miR-23a forward, 5'-CCTACTGTCGTCCCA AGACCT-3' and reverse, 5'-GGGGCTCGTGCAGAAGAA T-3'; U6 forward, 5'-ACAACTTTGGTATCGTGGAAGG-3' and reverse, 5'-GCCATCACGCCACAGTTTC-3'. GAPDH forward, 5'-GGAGCGAGATCCCTCCAAAAT-3' and reverse, 5'-GGCTGTTGTCATACTTCTCATGG-3'.

Cell culture and transfection. Human gastric mucosa epithelial line GES-1, and gastric cancer lines HGC-27, BGC-823, SGC-7901 and AGS were obtained from the Cell Bank of Central South University (Changsha, China). The cell lines were cultured in Dulbecco's modified Eagle's medium (DMEM) with $10 \%$ fetal bovine serum (FBS; both from Thermo Fisher Scientific, Inc.) at $37^{\circ} \mathrm{C}$ in a humidified incubator containing $5 \% \mathrm{CO}_{2}$. Cell transfection was conducted using Lipofectamine ${ }^{\circledR} 2000$ (Thermo Fisher Scientific, Inc.), according to the manufacturer's protocol. AGS cells were transfected with $100 \mathrm{nM}$ of scrambled miR mimic (miR-NC; Guangzhou Fulengen Co., Ltd., Guangzhou, China.), miR-23a mimic (miR-23a; Guangzhou Fulengen Co., Ltd.), miR-23a inhibitor (Fulengen), negative control inhibitor (NC inhibitor; Guangzhou Fulengen Co., Ltd.), co-transfected with miR-23a inhibitor and non-specific siRNA (miR-23a in+siNC; OriGene Technologies, Inc., Beijing, China.) or co-transfected with miR-23a inhibitor and SPRY2-specific siRNA (miR-23a in+siSPRY2; OriGene Technologies, Inc.). Following transfection for $48 \mathrm{~h}$, the following experiments were performed.

Western blot analysis. Cells were lysed in RIPA buffer (Thermo Fisher Scientific, Inc.), and the protein was extracted and quantified using a BCA Protein Assay kit (Pierce; Thermo Fisher Scientific, Inc.). Proteins $(50 \mu \mathrm{g})$ were separated with $10 \%$ SDS-PAGE and transferred onto a polyvinylidene difluoride (PVDF) membrane (Thermo Fisher Scientific, Inc.). The membrane was incubated with TBS with Tween ${ }^{\circledR} 20$ containing $5 \%$ non-fat milk at room temperature for $3 \mathrm{~h}$, and then with rabbit anti-mouse SPRY2 (dilution, 1:50; cat no. ab85670), total-ERK (dilution, 1:100; cat no. ab196883), phospho-ERK (dilution, 1:100; cat no. ab214362) or GAPDH antibodies (dilution, 1:100; cat no. ab9485; all from Abcam, Cambridge, MA, USA) at room temperature for $3 \mathrm{~h}$. Following washing with PBS with Tween 20 three times, the PVDF membrane was incubated with horseradish peroxidase-conjugated goat anti-rabbit secondary antibody (dilution, 1:10,000; cat no. ab6721; Abcam) at room temperature for $1 \mathrm{~h}$. Chemiluminescence detection was performed using a Pierce ${ }^{\mathrm{TM}}$ Fast Western Blot kit (Pierce; Thermo Fisher Scientific, Inc.). The relative protein expression was analyzed using Image-Pro Plus software version 6.0 (Media Cybernetics, Inc., Rockville, MD, USA), represented as the density ratio vs. GAPDH.

MTT assay. AGS cells in $100 \mu 1$ DMEM containing $0.5 \mathrm{~g} / 1 \mathrm{MTT}$ (Thermo Fisher Scientific, Inc.) were seeded in a 96-well plate at a density of $1 \times 10^{4}$ cells/well. Following incubation at $37^{\circ} \mathrm{C}$ for $0,24,48$ or $72 \mathrm{~h}, 50 \mu \mathrm{l}$ DMSO (Thermo Fisher Scientific, Inc.) was added. Following incubation at $37^{\circ} \mathrm{C}$ for $10 \mathrm{~min}$, the wavelength of $570 \mathrm{~nm}$ for each sample was measured using the Tecan Infinite ${ }^{\circledR}$ M200 plate reader (Tecan Group, Ltd., Mannedorf, Switzerland).

Wound healing assay. A wound healing assay was conducted to determine the cell migratory capacity. AGS cells were cultured to full confluence, and wounds of $\sim 1 \mathrm{~mm}$ width were created with a plastic scriber. Then, cells were washed once using PBS. Prior to migration, AGS cells were photographed under an inverted microscope (magnification, x40; Olympus Corporation, Tokyo, Japan) as the negative control. Following being cultured at $37^{\circ} \mathrm{C}$ with $5 \% \mathrm{CO}_{2}$ for $48 \mathrm{~h}$, AGS cells were observed under an inverted microscope (magnification, $\mathrm{x} 40$; Olympus Corporation). This experiment was repeated for 3 times.

Transwell assay. A Cell Invasion Assay kit (Chemicon International, Inc., Temecula, CA, USA), with an adhesion matrix supplied in the kit, was used to perform the Transwell assay, according to the manufacturer's guidelines. An AGS cell suspension was prepared in $300 \mu$ l DMEM containing 
Table I. Association between miR-23a expression and clinicopathological characteristics of patients with gastric cancer.

miR-23a expression

\begin{tabular}{|c|c|c|c|}
\hline \multirow[b]{2}{*}{ Variables } & & \multirow[b]{2}{*}{ P-value } \\
\hline & High $(n=41)$ & Low $(n=37)$ & \\
\hline Age, years & & & 0.820 \\
\hline$>65$ & 24 & 20 & \\
\hline$<65$ & 17 & 17 & \\
\hline Sex & & & 0.360 \\
\hline Male & 26 & 19 & \\
\hline Female & 15 & 18 & \\
\hline T stage & & & 0.251 \\
\hline $\mathrm{T} 1-2$ & 14 & 18 & \\
\hline T3-4 & 27 & 19 & \\
\hline Lymph node metastasis & & & $0.001^{\mathrm{a}}$ \\
\hline Present & 29 & 12 & \\
\hline Absent & 12 & 25 & \\
\hline Distant metastasis & & & 0.091 \\
\hline Present & 8 & 2 & \\
\hline Absent & 33 & 35 & \\
\hline Clinical stage & & & $0.006^{\mathrm{a}}$ \\
\hline I-II & 13 & 24 & \\
\hline III-IV & 28 & 13 & \\
\hline
\end{tabular}

${ }^{\mathrm{a}} \mathrm{P} \leq 0.001 ; \mathrm{T}$, tumor; miR, microRNA.

50,000 AGS cells and was seeded in the upper chamber. Then, $500 \mu$ I DMEM with 10\% FBS was added into the lower chamber. Following incubation at $37^{\circ} \mathrm{C}$ for $24 \mathrm{~h}$, the non-invading AGS cells on the upper face of the membrane were scraped, while the invading AGS cells on the lower face of the membrane were stained with $0.5 \%$ crystal violet at room temperature for 20 min and counted under an inverted microscope (magnification, x200).

Bioinformatics analysis. The putative target genes of miR-23a were predicted using Targetscan software (www.targetscan.org/), in accordance with the manufacturer's protocol.

Luciferase reporter gene assay. The mutant type (MT) 3'-UTR of SPRY2A was constructed using a Quik-Change Site-Directed Mutagenesis kit (Agilent Technologies, Inc., Santa Clara, CA, USA), according to the manufacturer's protocol. The wild type (WT) or MT 3'-UTR of SPRY2 were then inserted into the psiCHECK2 vector (Promega Corporation, Madison, WI, USA). For the luciferase reporter gene assay, AGS cells were transfected using Lipofectamine ${ }^{\circledR} 2000$ (Invitrogen, Thermo Fisher Scientific, Inc.) with $100 \mathrm{nM}$ of WT SPRY2-3'-UTR or MT SPRY2-3'-UTR plasmid, with or without $100 \mathrm{nM}$ of miR-23a mimics, respectively. The luciferase activity was then measured at $48 \mathrm{~h}$ following transfection using the Dual-Luciferase Reporter assay system (Promega Corporation) on the Lmax Multiwell luminometer (Molecular Devices,
LLC, Sunnyvale, CA, USA). Renilla luciferase activity was normalized to firefly luciferase activity.

Statistical analysis. The data are expressed as the mean \pm standard deviation following at least three independent experiments. Statistical analysis of differences was performed via one-way analysis of variance and Turkey's post-hoc test for multiple groups or Student's t-test for two groups, using the SPSS version 20.0 software package (IBM Corp., Armonk, NY, USA). $\mathrm{P}<0.05$ was considered to indicate a statistically significant difference. A $\chi^{2}$ test was performed for Table I, with a log-rank test conducted on the Kaplan-Meier survival curve.

\section{Results}

Upregulation of miR-23a is associated with gastric cancer progression and poor prognosis of patients. In the present study, RT-qPCR was conducted first to examine the expression levels of miR-23a in gastric cancer. The data indicated that miR-23a was significantly upregulated in gastric cancer tissues compared with adjacent non-tumor tissues (Fig. 1A). Further investigation revealed that the increased expression of miR-23a was significantly associated with node metastasis and advanced clinical stage (Table I). The results indicated that miR-23a may contribute to gastric cancer growth and metastasis. Furthermore, the patients with gastric cancer with high miR-23a expression demonstrated significantly reduced survival time, compared with those with low miR-23a levels (Fig. 1B); therefore, miR-23a may be used as a potential predicator for the prognosis of patients with gastric cancer.

Knockdown of miR-23a decreases AGS cell proliferation, migration and invasion. The miR-23a levels in gastric cancer cell lines, including HGC-27, BGC-823, SGC-7901 and AGS cells, were further examined. The data indicated that miR-23a was also significantly upregulated in gastric cancer cell lines compared with normal gastric epithelial GES-1 cells (Fig. 2A). AGS cells were used to conduct in vitro experiments. As miR-23a was demonstrated to be significantly upregulated in gastric cancer, AGS cells were transfected with a miR-23a inhibitor to knockdown its expression. Following transfection, the miR-23a levels were significantly reduced in the miR-23a inhibitor group compared with the NC inhibitor group (Fig. 2B). The MTT assay data further demonstrated that knockdown of miR-23a caused a significant decrease in AGS cell proliferation after $48 \mathrm{~h}$ (Fig. 2C). The results of a wound healing assay and Transwell assay demonstrated that inhibition of miR-23a significantly suppressed AGS cell migration and invasion (Fig. 2D and E). According to this data, it was indicated that miR-23a serves a promoter role in gastric cancer growth and metastasis, and may be used as a potential therapeutic target.

SPRY2, which is downregulated in gastric cancer, is a target of miR-23a in AGS cells. Bioinformatics analysis was conducted to predict the target of miR-23a, which indicated SPRY2 to be a potential target (Fig. 3A). To further confirm this target association, the luciferase reporter gene plasmid containing the WT or MT of SPRY2-3'-UTR (Fig. 3B) was constructed. Following this, a luciferase reporter assay in AGS cells was performed. 

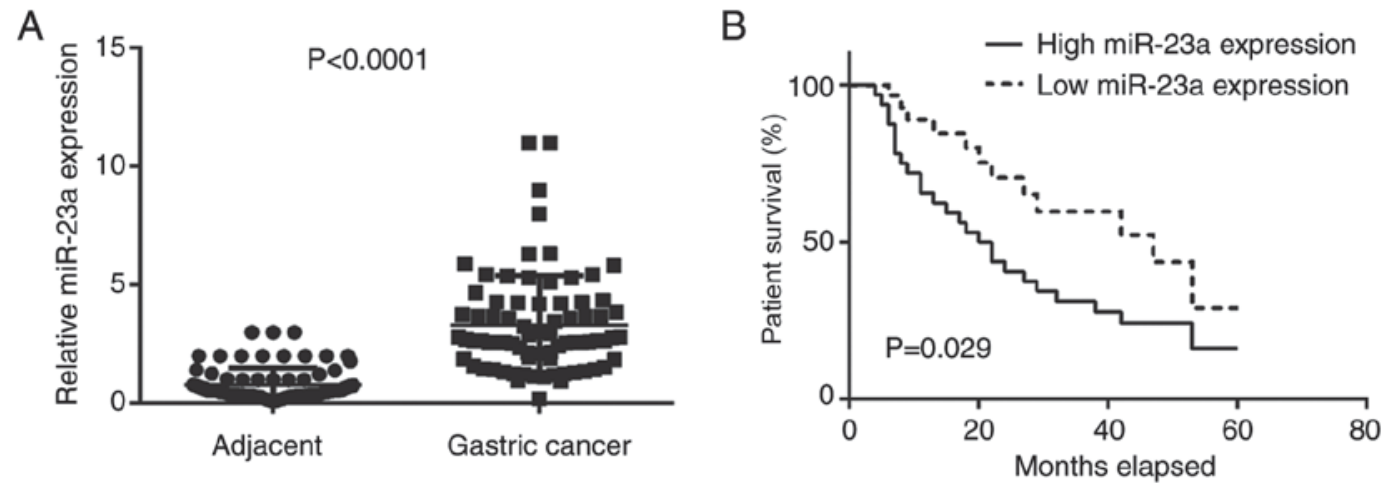

Figure 1. (A) Reverse transcription-quantitative polymerase chain reaction was conducted to examine the miR-23a levels in gastric cancer tissues and adjacent tissues $(\mathrm{P}<0.0001)$. (B) Patients with gastric cancer with high miR-23a expression demonstrated reduced survival time, when compared with those with low miR-23a levels $(\mathrm{P}=0.029)$. miR, microRNA.
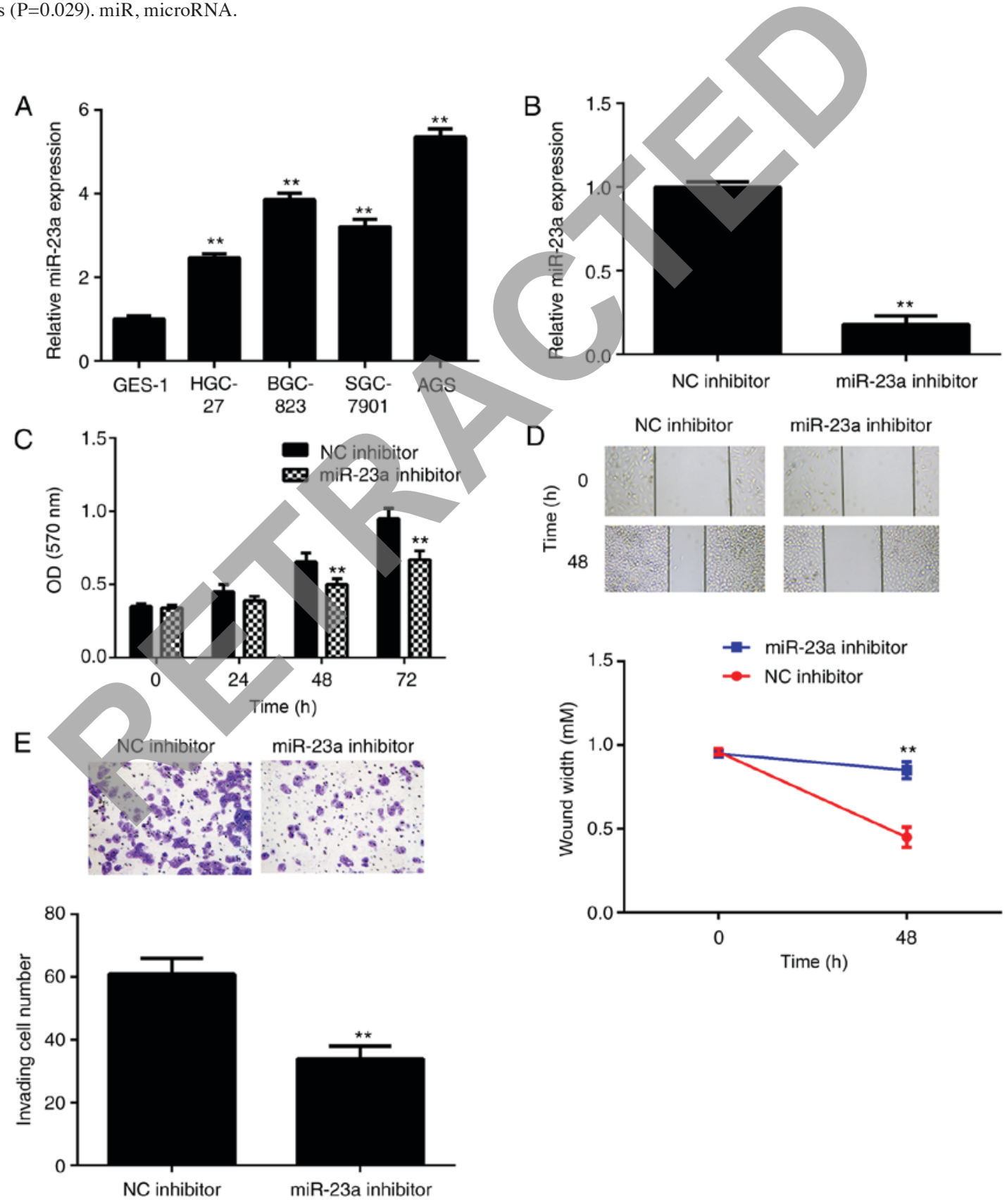

Figure 2. (A) RT-qPCR was conducted to examine the miR-23a levels in gastric cancer cell lines, compared with normal gastric mucosa epithelial GES-1 cells. ${ }^{* *} \mathrm{P}<0.01$ vs. GES-1. Following this, AGS cells were transfected with miR-23a inhibitor or NC inhibitor. (B) RT-qPCR was conducted to examine the miR-23a levels. (C) MTT assay, (D) wound healing assay and (E) Transwell assay were conducted to examine cell proliferation, migration and invasion, respectively. ${ }^{* *} \mathrm{P}<0.01$ vs. NC inhibitor. The magnifications for the wound healing and Transwell assay images were $\mathrm{x} 40$ and x200, respectively. RT-qPCR, reverse transcription-quantitative polymerase chain reaction; NC, negative control; miR, microRNA; OD, optical density. 
A Position 458-465 of SPRY2-3'-UTR 5'...GUACAUUCGgAaGAAUGUGAA...3'

miR-23a 3'...CCUUUAGGGACCGUUACACUA...5'

B

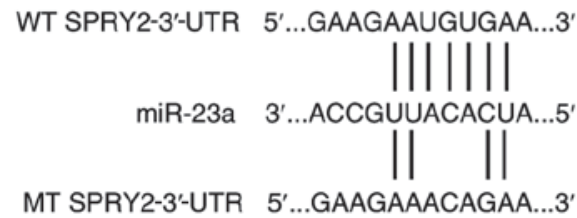

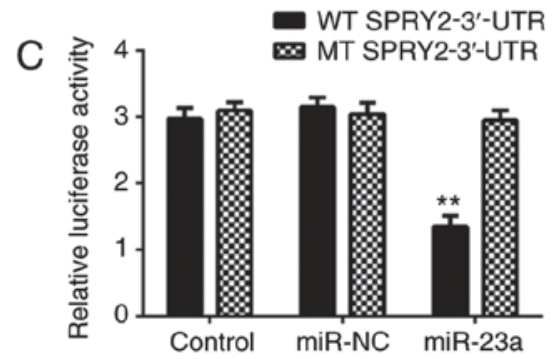

$\mathrm{E}$

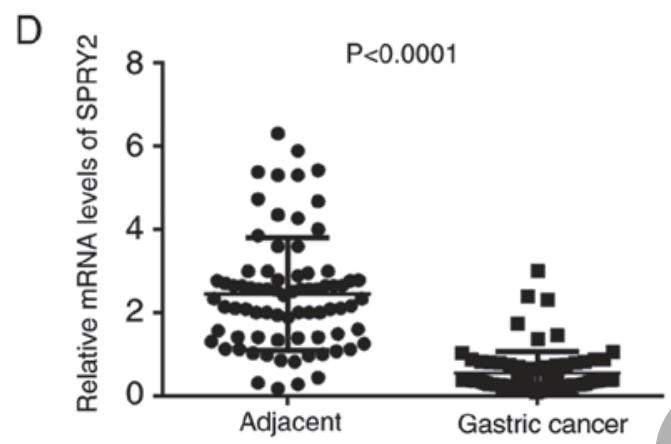

$\mathrm{F}$

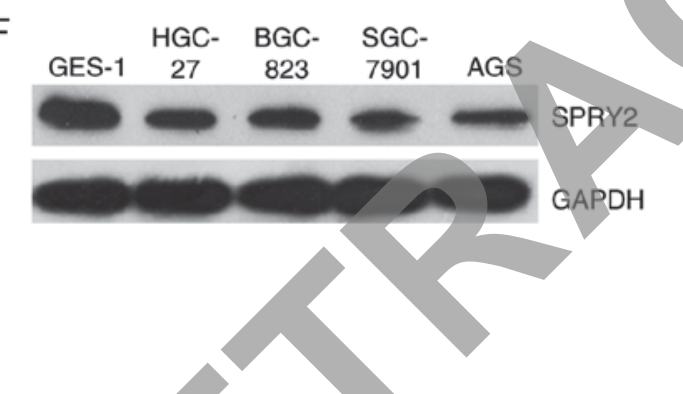

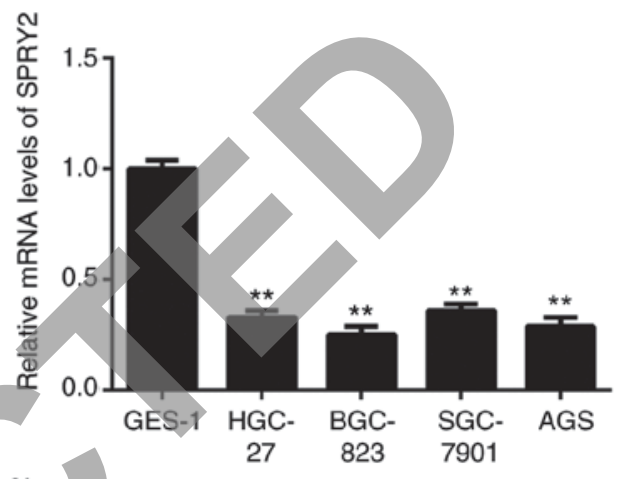

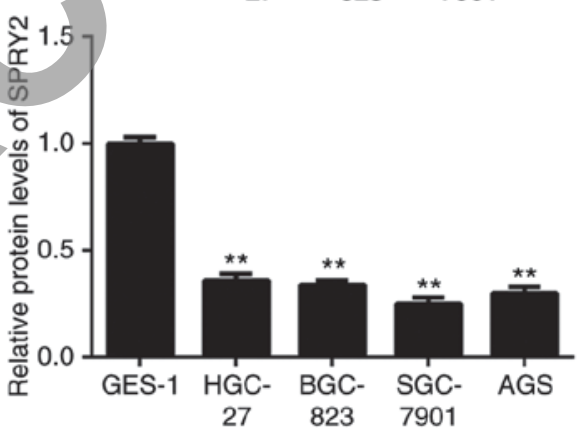

Figure 3. (A) SPRY2 is predicted to be a potential target of miR-23a. (B) The luciferase reporter gene plasmids containing the wild type (WT) and mutant type (MT) of SPRY2-3'-UTR were generated. (C) Luciferase reporter gene assay data indicated that the luciferase activity was reduced in AGS cells co-transfected with miR-23a mimics and WT 3-UTR of SPRY2 reporter plasmid, but unaltered in cells co-transfected with miR-23a mimics and MT SPRY2-3'-UTR plasmid. ${ }^{* *} \mathrm{P}<0.01$ vs. Control. (D) RT-qPCR was conducted to examine the SPRY2 mRNA levels in gastric cancer tissues and matched adjacent tissues (P<0.0001). ${ }^{* *} \mathrm{P}<0.01$ vs. Adjacent. (E) RT-qPCR and (F) western blot analysis was conducted to examine the mRNA and protein levels of SPRY2 in gastric cancer cell lines, compared with normal gastric mucosa epithelial GES-1 cells. ${ }^{* *} \mathrm{P}<0.01$ vs. GES-1. SPRY2, sprouty homolog 2; miR, microRNA; NC, negative control; UTR, untranslated region, MT, mutated type; WT, wild type.

The luciferase activity was significantly reduced following co-transfection with the miR-23a mimics and WT 3'-UTR of SPRY2 plasmid, but unaltered following co-transfection with the miR-23a mimics and MT SPRY2-3'-UTR plasmid (Fig. 3C). This data demonstrated that miR-23a directly binds to the 3'-UTR of SPRY2 mRNA in gastric cancer AGS cells.

Following this, the expression levels of SPRY2 in gastric cancer tissues and cell lines were investigated. As depicted in Fig. 3D, the mRNA levels of SPRY2 were significantly reduced in gastric cancer tissues compared with normal adjacent tissues. Similarly, the mRNA and protein levels of SPRY2 were downregulated in gastric cancer cell lines (Fig. 3E and F).

The effects of miR-23a on the protein expression of SPRY2 in AGS cells were studied. The data indicated that knockdown of miR-23a enhanced the mRNA and protein expression of SPRY2 in AGS cells (Fig. 4A and B). To further confirm this data, AGS cells were transfected with the miR-23a mimic to increase its expression. Following transfection, the miR-23a levels were upregulated in the miR-23a group compared with the miR-NC group (Fig. 4C). The upregulation of miR-23a reduced the mRNA and protein levels of SPRY2 (Fig. 4D and E); therefore, miR-23a negatively regulates the expression of SPRY2 in AGS cells.

SPRY2 is involved in the miR-23a-mediated AGS cells. Based on the aforementioned data, it was speculated that SPRY2 may be involved in the miR-23a-mediated AGS cells. To clarify this speculation, AGS cells were co-transfected with the miR-23a inhibitor and SPRY2-specific siRNA plasmid, or co-transfected with the miR-23a inhibitor and non-specific siRNA in the control group. Following transfection, the mRNA and protein levels of SPRY2 were significantly downregulated 
A

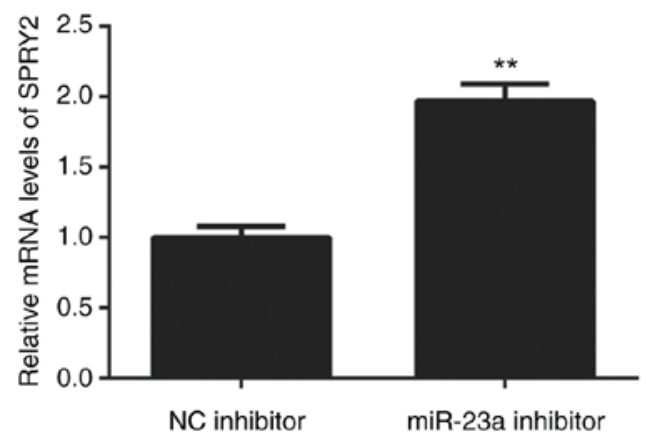

C

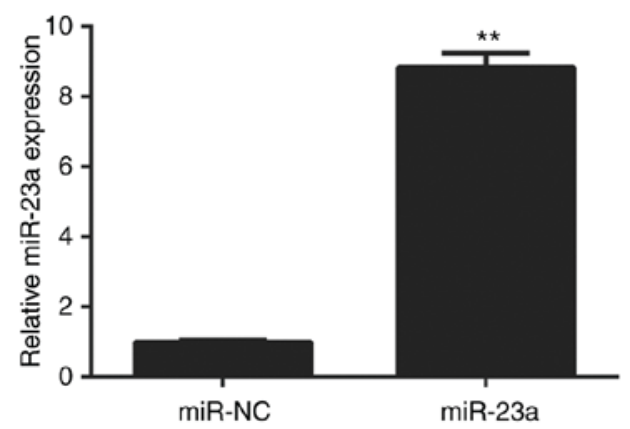

E

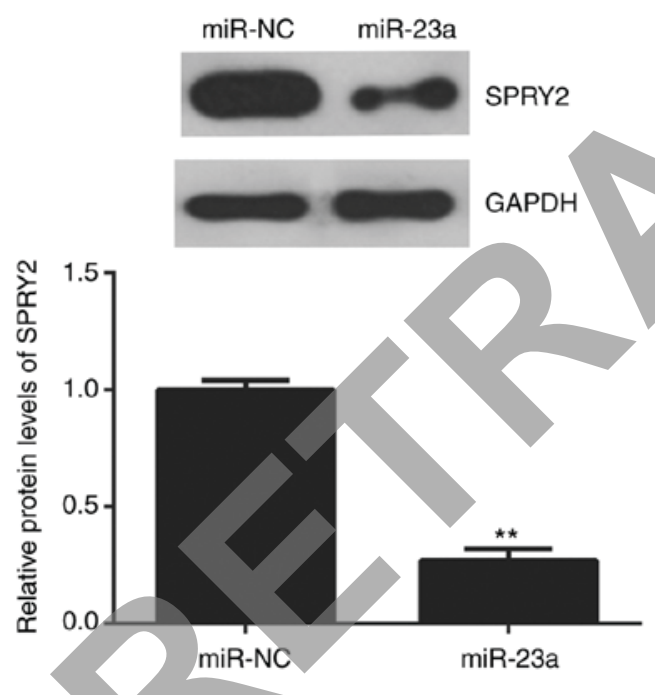

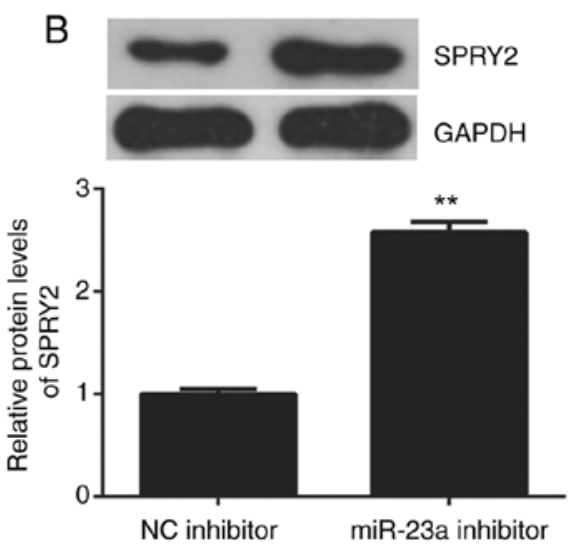

D

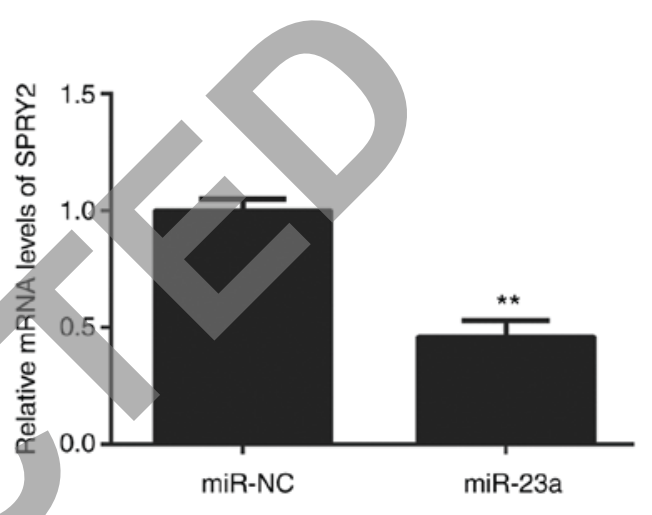

Figure 4. (A) RT-qPCR and (B) western blot analysis were conducted to examine the mRNA and protein levels in AGS cells transfected with miR-23a inhibitor or NC inhibitor. ${ }^{* * *} \mathrm{P}<0.01$ vs. NC inhibitor. Following this, AGS cells were transfected with miR-23a mimic or scramble miR mimic, miR-NC. Following transfection, (C) RT-qPCR was conducted to examine the miR-23a levels. Then, (D) RT-qPCR and (E) western blot analysis were conducted to determine the mRNA and protein levels of SPRY2. (C-E) "** $\mathrm{P}<0.01$ vs. miR-NC. NC, negative control; RT-qPCR, reverse transcription-quantitative polymerase chain reaction; SPRY2, sprouty homolog 2; miR, microRNA.

in the miR-23a in+siSPRY2 group compared with the miR-23a in+siNC group (Fig. 5A and B). Furthermore, it was demonstrated that the proliferation, migration and invasion of AGS cells were significantly upregulated in the miR-23a inhibitor+SPRY2 siRNA group compared with the miR-23a inhibitor+NC siRNA group (Fig. 5C-E). The results indicated that the suppressive effect of miR-23a knockdown on AGS cells may occur via the upregulation of SPRY2.

Activity of ERK signaling is mediated by miR-23a and SPRY2 in AGS cells. SPRY2 has been reported to have inhibitory effects on the activity of ERK signaling, which is essential for cancer cell growth and metastasis (23). The activity of
ERK signaling was thus studied. As depicted in Fig. 6A, knockdown of miR-23a reduced the phosphorylation levels of the ERK protein; however, the phosphorylation levels of the ERK protein were increased in the miR-23a in+siSPRY2 group compared with the miR-23a in+siNC group (Fig. 6B); therefore, it is suggested that miR-23a promotes the activity of ERK signaling via targeting SPRY2.

\section{Discussion}

The regulatory mechanism underlying miR-23a in gastric cancer remains unclear. In the present study, it was demonstrated that miR-23a was significantly upregulated in gastric 
A
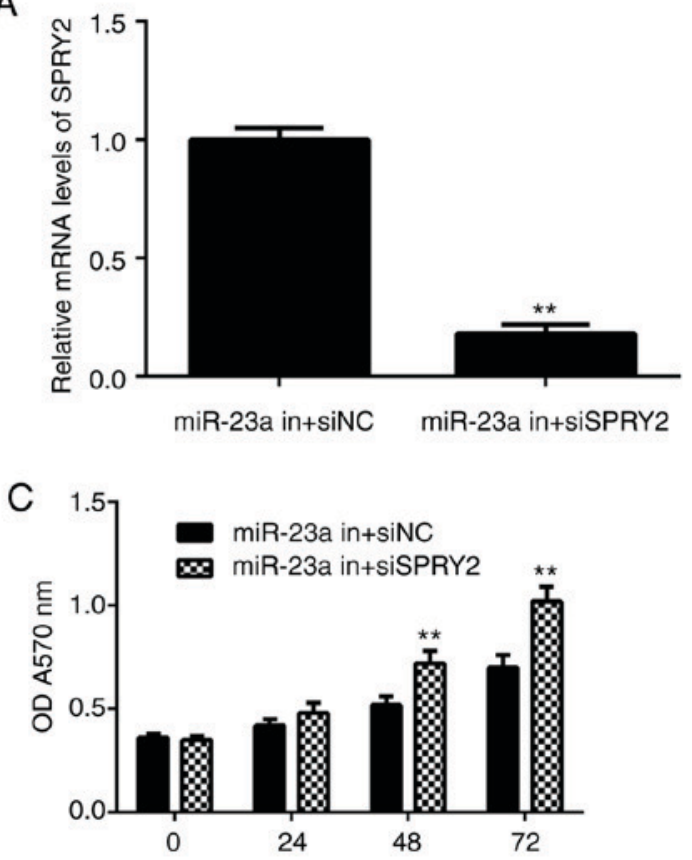

E miR-23a in+siNC miR-23a in+siSPRY2
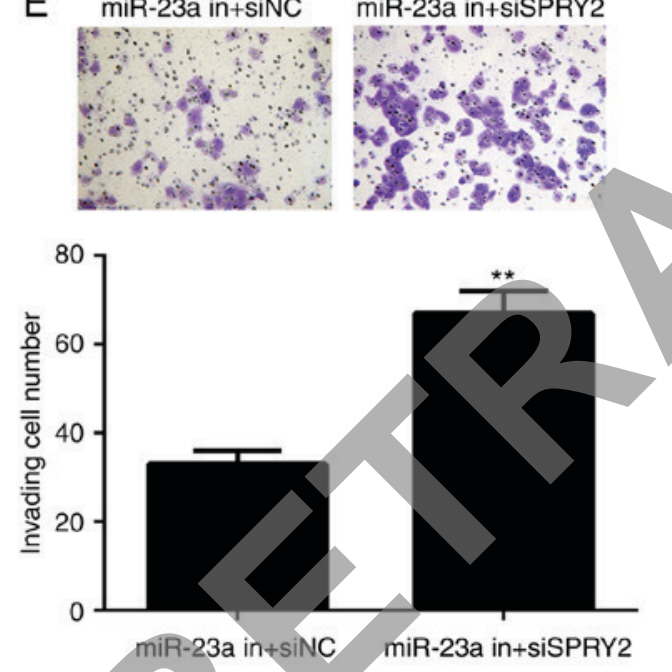

B
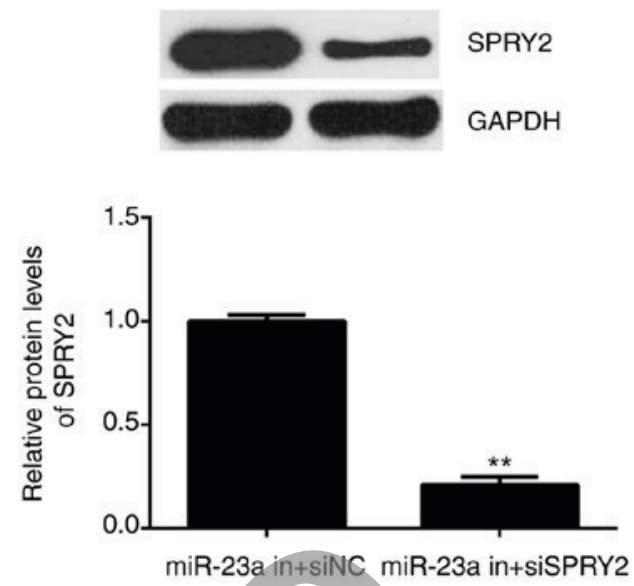

D

miR-23a in+siNC miR-23a in+siSPRY2

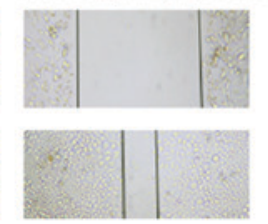

$\rightarrow$ miR-23a in+siSPRY2

$\rightarrow$ miR-23a in+sinc

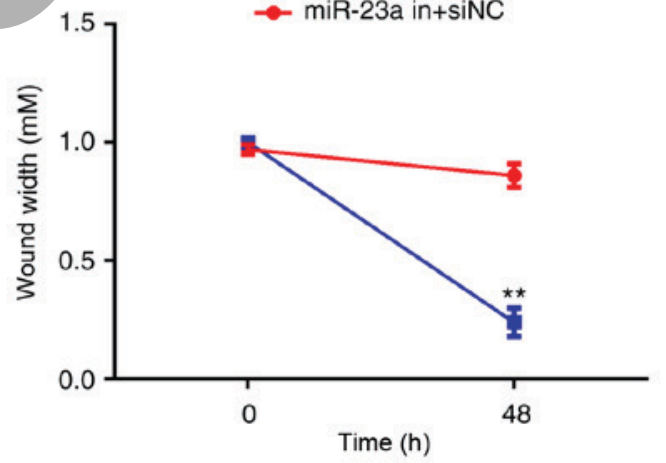

Figure 5. AGS cells were co-transfected with miR-23a inhibitor and SPRY2-specific siRNA, miR-23a in+siSPRY2, or co-transfected with miR-23a inhibitor and non-specific siRNA, miR-23a in+siNC. Following this, (A) reverse transcription-quantitative polymerase chain reaction and (B) western blot analysis were used to examine the mRNA and protein expression of SPRY2. (C) An MTT, (D) wound healing and (E) Transwell assay were conducted to examine cell proliferation, migration and invasion, respectively. ${ }^{* *} \mathrm{P}<0.01 \mathrm{vs}$. miR-23a in + siNC. The magnification for wound healing assay image was $\mathrm{x} 40$, for Transwell assay was x200. si, small interfering; miR, microRNA; NC, negative control; SPRY, sprouty homolog 2; in, inhibitor.

cancer tissues and cell lines, and its upregulation was significantly associated with cancer progression and poor prognosis of patients. Knockdown of miR-23a significantly decreased the proliferation, migration and invasion of AGS cells. SPRY2, which, in the present study, is significantly downregulated in gastric cancer tissues and cell lines, was identified as a target gene of miR-23a, and its expression was negatively regulated by miR-23a at the post-transcriptional levels in AGS cells. siRNA-induced SPRY2 inhibition reduced the suppressive effects of miR-23a downregulation in AGS cells. In addition, the activity of ERK signaling was inhibited by the miR-23a/SPRY2 knockdown in AGS cells.

It has been demonstrated that numerous miRs are deregulated and serve important roles in gastric cancer $(24,25)$. For example, miR-218 inhibits proliferation, migration and EMT of gastric cancer cells via targeting WAS protein family member 3 (9). miR-181b inhibits the glycolysis in gastric cancer cells via inhibiting the expression of hexokinase 2 (26). The present study demonstrated that the expression of miR-23a was significantly increased in gastric cancer tissues and cell lines, compared with adjacent non-tumor tissues or normal gastric epithelial cells, respectively. The results of the current study were consistent with previous studies $(15,27,28)$. Following this, it was determined that the increased expression of miR-23a was significantly associated with gastric cancer progression and the poor prognosis of patients. Similarly, Ma et al (29) demonstrated that the co-expression of miR-23a and miR-23b in gastric cancer tissues was significantly associated with advanced stage, lymph node metastasis and invasion, and it was an independent predictor for unfavorable 

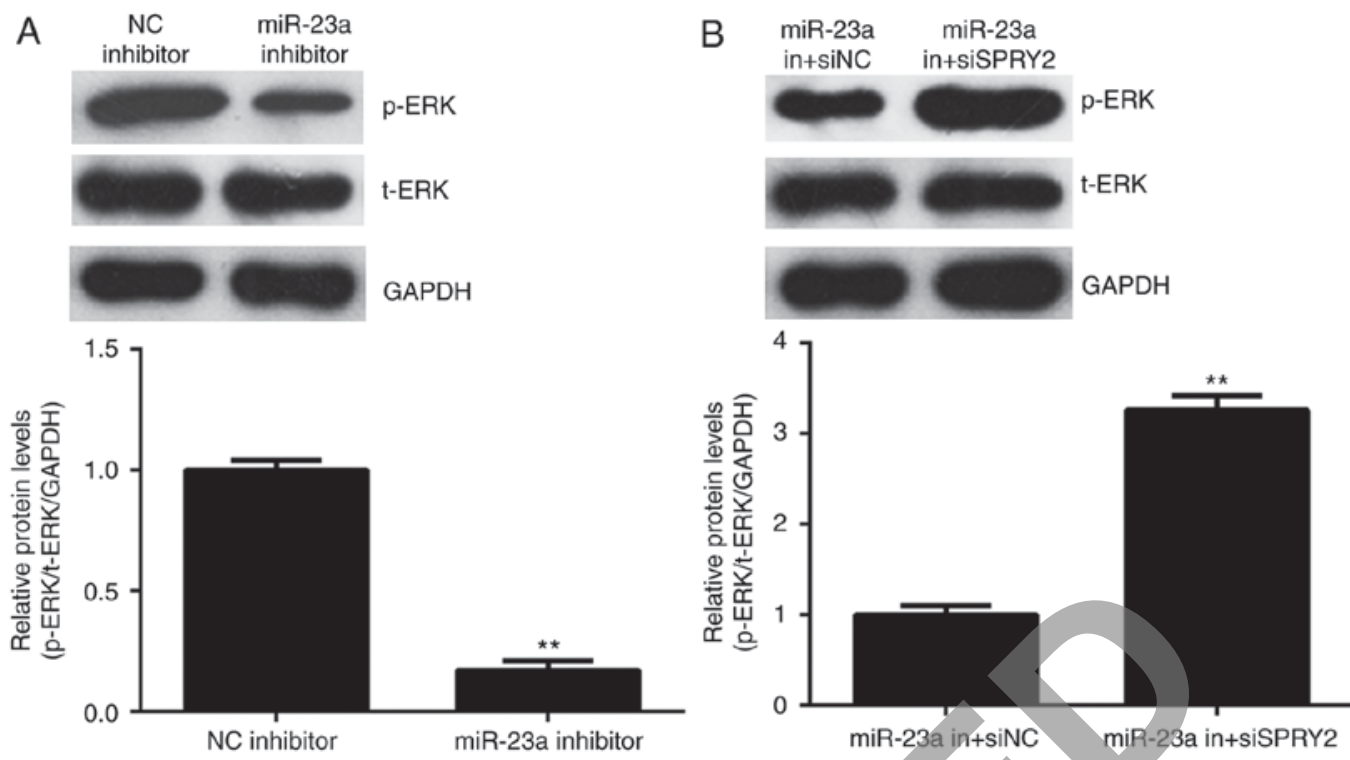

Figure 6. (A) Western blot analysis was used to examine the levels of the p-ERK and t-ERK protein levels in AGS cells transfected with miR-23a inhibitor or negative control (NC) inhibitor. ${ }^{* *} \mathrm{P}<0.01$ vs. NC inhibitor. (B) Western blot analysis was used to examine levels of the p-ERK protein and t-ERK protein levels in AGS cells co-transfected with miR-23a inhibitor and SPRY2-specific siRNA (miR-23a in+siSPRY2), or co-transfected with miR-23a inhibitor and non-specific siRNA (miR-23a in+siNC). ${ }^{* *} \mathrm{P}<0.01$ vs. miR-23a in+siNC. ERK, extracellular signal-regulated kinases; NC, negative control; miR, microRNA; si, small interfering; SPRY2, sprouty homolog 2; p, phospho; t, total; in, inhibitor.

overall survival time. Furthermore, it was demonstrated that knockdown of miR-23a could was able to reduce the proliferation, migration and invasion of AGS cells in the current study. Additionally, Zhu et al (30) demonstrated that inhibition of miR-23a was able to inhibit the proliferation and invasion of gastric cancer MGC803 cells; however, the underlying molecular regulatory mechanism of miR-23a in gastric cancer migration, and invasion remains unknown.

It has been well established that SPRY2 is an important tumor suppressor, and is regulated by a number of miRs (19-21). For instance, SPRY2 is able to inhibit the phosphorylation of ERK downstream of receptor tyrosine kinase signaling, which is important for tumor growth, metastasis and drug-resistance in human cancer types $(20,21)$. Additionally, miR-27b promotes cell invasion of glioma U251 cells via targeting SPRY2 (19). miR-21 upregulates the ERK-mitogen-activated protein kinase signaling pathway via targeting SPRY2 during human mesenchymal stem cell differentiation (31). In the present study, it was demonstrated that miR-23a was able to directly target SPRY2, which was involved in the miR-23a-mediated proliferation, migration and invasion of AGS cells. The downstream ERK signaling in AGS cells was further studied. The results indicated that the knockdown of miR-23a significantly inhibited the activity of ERK signaling in AGS cells, which was reversed via SPRY2 downregulation. This data indicated that miR-23a was able to activate the ERK signaling via the inhibition of SPRY2, and thus promote the malignant phenotypes of AGS cells. Similarly, a previous study also indicated that miR-27a was able to promote the migration and invasion of gastric cancer cells via inhibiting the expression of SPRY2, and thus activating ERK signaling (32).

To the best of our knowledge, this is the first study to report that miR-23a promoted the proliferation, migration and invasion of gastric cancer cells via targeting the tumor suppressor SPRY2, and thus upregulating the activity of ERK signaling.
Therefore, the results of the present study suggest that miR-23a may be a potential therapeutic target for gastric cancer. In addition, further investigations are required to explore the regulatory effects of miR-23a/SPRY2 in gastric cancer cell in vivo.

\section{Acknowledgements}

Not applicable.

\section{Funding}

No funding was received.

\section{Availability of data and materials}

All data generated or analyzed during this study are included in this published article.

\section{Author's contributions}

YL collected clinical tissues, did the clinical experiments, wrote the manuscript and submitted it. HC designed the present study and revised this manuscript. PS, TC, LC, JY and $\mathrm{BJ}$ performed the in vitro experiments.

\section{Ethics approval and consent to participate}

The present study was approved by the Ethical Committee of The Third Xiangya Hospital. Written informed consent was obtained from all patients involved in the present study.

\section{Consent for publication}

Written informed consent was obtained from all participants for publication of the present study. 


\section{Competing interests}

The authors declare that they have no competing interests.

\section{References}

1. Siegel RL, Miller KD and Jemal A: Cancer statistics, 2015. CA Cancer J Clin 65: 5-29, 2015

2. Torre LA, Bray F, Siegel RL, Ferlay J, Lortet-Tieulent J and Jemal A: Global cancer statistics, 2012. CA Cancer J Clin 65 87-108, 2015.

3. Piazuelo MB and Correa P: Gastric cancer: Overview. Colomb Med (Cali) 44: 192-201, 2013.

4. Ambros V: The functions of animal microRNAs. Nature 431: 350-355, 2004.

5. Calin GA, Sevignani C, Dumitru CD, Hyslop T, Noch E, Yendamuri S, Shimizu M, Rattan S, Bullrich F, Negrini M and Croce CM: Human microRNA genes are frequently located at fragile sites and genomic regions involved in cancers. Proc Natl Acad Sci USA 101: 2999-3004, 2004.

6. John B, Enright AJ, Aravin A, Tuschl T, Sander C and Marks DS: Human MicroRNA targets. PLoS Biol 2: e363, 2004.

7. Zhang X, Cai D, Meng L and Wang B: MicroRNA-124 inhibits proliferation, invasion, migration and epithelial-mesenchymal transition of cervical carcinoma cells by targeting astrocyte-elevated gene-1. Oncol Rep 36: 2321-2328, 2016.

8. Zhu K, He Y, Xia C, Yan J, Hou J, Kong D, Yang Y and Zheng G: MicroRNA-15a inhibits proliferation and induces apoptosis in CNE1 nasopharyngeal carcinoma cells. Oncol Res 24: 145-151, 2016.

9. Wang G, Fu Y, Liu G, Ye Y and Zhang X: miR-218 inhibits proliferation, migration, and EMT of gastric cancer cells by targeting WASF3. Oncol Res 25: 355-364, 2017.

10. Lv H, Zhang Z, Wang Y, Li C, Gong W and Wang X: MicroRNA-92a promotes colorectal cancer cell growth and migration by inhibiting KLF4. Oncol Res 23: 283-290, 2016

11. Liu X, Li J, Yu Z, Sun R and Kan Q: MiR-935 promotes cancer cell proliferation and migration by targeting SOX7. Oncol Res 25: 427-435, 2017.

12. Li X, Li Y and Lu H: MiR-1193 suppresses proliferation and invasion of human breast cancer cells through directly targeting IGF2BP2. Oncol Res 25: 579-585, 2017.

13. Liu P, Wang C, Ma C, Wu Q, Zhang W and Lao G: MicroRNA-23a regulates epithelial-to-mesenchymal transition in endometrial endometrioid adenocarcinoma by targeting SMAD3. Cancer Cell Int 16: 67, 2016.

14. He Y, Meng C, Shao Z, Wang H and Yang S: MiR-23a functions as a tumor suppressor in osteosarcoma. Cell Physiol Biochem 34: 1485-1496, 2014.

15. Zhu LH, Liu T, Tang H, Tian RQ, Su C, Liu M and Li X: MicroRNA-23a promotes the growth of gastric adenocarcinoma cell line MGC803 and downregulates interleukin-6 receptor. FEBS J 277: 3726-3734, 2010.

16. Liu X, Liu Q, Fan Y, Wang S, Liu X, Zhu L, Liu M and Tang H: Downregulation of PPP2R5E expression by miR-23a suppresses apoptosis to facilitate the growth of gastric cancer cells. FEBS Lett 588: 3160-3169, 2014.

17. Lim J, Wong ES, Ong SH, Yusoff P, Low BC and Guy GR: Sprouty proteins are targeted to membrane ruffles upon growth factor receptor tyrosine kinase activation. Identification of a novel translocation domain. J Biol Chem 275: 32837-32845, 2000.
18. Lim J, Yusoff P, Wong ES, Chandramouli S, Lao DH, Fong CW and Guy GR: The cysteine-rich sprouty translocation domain targets mitogen-activated protein kinase inhibitory proteins to phosphatidylinositol 4,5-bisphosphate in plasma membranes. Mol Cell Biol 22: 7953-7966, 2002.

19. Liu C, Liang S, Xiao S, Lin Q, Chen X, Wu Y and Fu J: MicroRNA-27b inhibits Spry2 expression and promotes cell invasion in glioma U251 cells. Oncol Lett 9: 1393-1397, 2015.

20. Chandramouli S, Yu CY, Yusoff P, Lao DH, Leong HF, Mizuno K and Guy GR: Tesk1 interacts with Spry2 to abrogate its inhibition of ERK phosphorylation downstream of receptor tyrosine kinase signaling. J Biol Chem 283: 1679-1691, 2008.

21. Wu G, Qin XQ, Guo JJ, Li TY and Chen JH: AKT/ERK activation is associated with gastric cancer cell resistance to paclitaxel. Int J Clin Exp Pathol 7: 1449-1458, 2014.

22. Livak KJ and Schmittgen TD: Analysis of relative gene expression data using real-time quantitative PCR and the 2(-Dalta Dalta C (T)) method. Methods 25: 402-408,2001.

23. Yim DG, Ghosh S, Guy GR and Virshup DM: Casein kinase 1 regulates Sprouty2 in FGF-ERK signaling. Oncogene 34: 474-484, 2015.

24. Deng J, Lei W, Xiang X, Zhang L, Yu F, Chen J, Feng M and Xiong J: MicroRNA-506 inhibits gastric cancer proliferation and invasion by directly targetìng Yap1. Tumour Biol 36: 6823-6831, 2015.

25. Lu WD, Zuo Y, Xu Z and Zhang M: MiR-19a promotes epithelial-mesenchymal transition through PI3K/AKT pathway in gastric cancer. World J Gastroenterol 21: 4564-4573, 2015.

26. Li QL, Yang Y, Zhang L, Chen H, Pan D and Xie WJ: MicroRNA-181b inhibits glycolysis in gastric cancer cells via targeting hexokinase 2 gene. Cancer Biomark 17: 75-81, 2016.

27. Li X, Zhang Y, Zhang H, Liu X, Gong T, Li M, Sun L, Ji G, Shi Y, Han Z, et al: miRNA-223 promotes gastric cancer invasion and metastasis by targeting tumor suppressor EPB41L3. Mol Cancer Res 9: 824-833, 2011.

28. An J, Pan Y, Yan Z, Li W, Cui J, Yuan J, Tian L, Xing R and Lu Y: MiR-23a in amplified 19p13.13 loci targets metallothionein 2A and promotes growth in gastric cancer cells. J Cell Biochem 114: 2160-2169, 2013.

29. Ma G, Dai W, Sang A, Yang X and Gao C: Upregulation of microRNA-23a/b promotes tumor progression and confers poor prognosis in patients with gastric cancer. Int J Clin Exp Pathol 7: 8833-8840, 2014.

30. Zhu L, Tian J, Chen L, Wang M, Xiong Y,Zhang G, Li S and Yuan L: Effect of blocking endogenous miR-23a on the proliferation and invasion in gastric adenocarcinoma cell line MGC803. Nan Fang Yi Ke Da Xue Xue Bao 33: 678-683, 2013 (In Chinese).

31. Mei Y, Bian C, Li J, Du Z, Zhou H, Yang Z and Zhao RC: miR-21 modulates the ERK-MAPK signaling pathway by regulating SPRY2 expression during human mesenchymal stem cell differentiation. J Cell Biochem 114: 1374-1384, 2013.

32. Jiang J, Yi B, Qin C, Ding S and Cao W: Upregulation of microRNA27b contributes to the migration and invasion of gastric cancer cells via the inhibition of sprouty2-mediated ERK signaling. Mol Med Rep 13: 2267-2272, 2016.

This work is licensed under a Creative Commons Attribution-NonCommercial-NoDerivatives 4.0 International (CC BY-NC-ND 4.0) License. 